\title{
Epidemiological characteristics of carbapenem-resistant Enterobacteriaceae collected from 17 hospitals in Nanjing district of China
}

Hui Zhou ${ }^{1 \dagger}$, Kui Zhang ${ }^{1+}$, Wei Chen ${ }^{2}$, Junhao Chen ${ }^{1}$, Jie Zheng ${ }^{1}$, Chang Liu', Li Cheng ${ }^{1}$, Wanqing Zhou', Han Shen ${ }^{1 *}$ and Xiaoli Cao ${ }^{1 *}$ (D)

\begin{abstract}
Objective: In total, 97 carbapenem-resistant Enterobacteriaceae (CRE) were collected from 17 hospitals located in Nanjing, Southeast China, and analyzed for epidemiological characteristics.

Methods: Antimicrobial susceptibility was determined; followed by determination of the prevalence of resistance determinants, including extended-spectrum $\beta$-lactamase (ESBLs), plasmid-mediated AmpC enzyme (pAmpCs), plasmid-mediated quinolone resistance genes (PMQRs), fosfomycin resistance gene and exogenously acquired $16 \mathrm{~S}$ rRNA methyltransferase (16S-RMTase) using PCR and DNA sequencing. The sequence types (STs) of CRE were determined by multi-locus sequence typing (MLST). The plasmid profiles were detected by PCR-based replicon typing (PBRT).

Results: All the CRE strains displayed high $\mathrm{MIC}_{50}$ and $\mathrm{MIC}_{90}$ for nearly all clinical available antibiotics, except for aztreonam/avibactam, minocycline, ceftazidime/avibactam, tigecycline, and colistin. KPC-2 (79.4\%) and NDM (19.6\%) were the main carbapenemases, CTX-M (76.3\%) and SHV (60.8\%) were the predominant ESBLs. In addition, oqXAB (70.1\%) and anr (63.9\%) were the major PMQRs; rmtB (47.4\%) was the main 16S-RMTase; fosA (76.3\%) and fosA3 (37.1\%) were the fosfomycin resistance gene. PBRT analysis showed presence of IncR (66.0\%) and IncFII (64.9\%) replicon types in the majority of the isolates, followed by IncFIB (46.4\%) and IncX3 (16.5\%). The IncFII and IncR replicon-types were found mainly in K. pneumoniae (68.8\%), whereas the IncX3 replicons dominated in E. coli isolates (100.0\%). The three dominating MLST-types ST11, ST15 and ST268 comprised $68.0 \%$ of the $77 \mathrm{~K}$. pneumoniae. Seven distinct STs were identified among 8 E. coli.

Conclusions: The treatment for infections caused by CRE isolates is challenged by the presence of multiple resistance determinants and plasmid replicons. Our results highlighted the expansion of blaKPC-2 carrying $K$. pneumoniae ST11, the new emergency of single blaNDM-5 carrying K. oxytoca ST36, as well as blalMP-4 and blaNDM-1 co-carrying E. cloacae ST418, which alert us on the urgency for antimicrobial resistant surveillance, to prevent dissemination of these highly transmissible and dangerous lineages.
\end{abstract}

Keywords: CRE, Carbapenemase, Plasmid replicon, Sequence type, Klebsiella pneumoniae

*Correspondence: shenhan10366@sina.com; cao-xiao-li@163.com

${ }^{\dagger}$ Hui Zhou and Kui Zhang contributed equally to this work.

${ }^{1}$ Department of Laboratory Medicine, Nanjing Drum Tower Hospital, the

affiliated Hospital of Nanjing University Medical School, Zhongshan Road

321, Gulou District, Nanjing, Jiangsu, People's Republic of China

Full list of author information is available at the end of the article

(c) The Author(s). 2020 Open Access This article is distributed under the terms of the Creative Commons Attribution 4.0 International License (http://creativecommons.org/licenses/by/4.0/), which permits unrestricted use, distribution, and reproduction in any medium, provided you give appropriate credit to the original author(s) and the source, provide a link to the Creative Commons license, and indicate if changes were made. The Creative Commons Public Domain Dedication waiver (http://creativecommons.org/publicdomain/zero/1.0/) applies to the data made available in this article, unless otherwise stated. 


\section{Introduction}

With the wide use of carbapenem antibiotics in clinical therapy, carbapenem-resistant Enterobacteriaceae (CRE) has dramatically increased and become a serious public health issue [1]. CRE constitutes a large group of bacteria with different mechanisms for drug resistance [2]. Among them, carbapenem resistant Klebsiella pneumoniae (CRKP) accounts for approximately $60 \%$, followed by Escherichia coli and Enterobacter cloacae [3, 4]. Epidemiological studies have shown that production of carbapenemase is the main mechanism for carbapenem resistance [5], with blaKPC and blaNDM being the most prevalent ones in the CRKP and carbapenem resistant $E$. coli (CREC), respectively [6, 7]. Recently, the cooccurrence of multiple resistance determinants, even more than 2 carbapenemases in one strain, has been frequently reported $[8,9]$, alerting us on the importance of resistance surveillance, since the resistant determinants are mainly plasmid-borne with highly transmissible nature $[9,10]$. Additionally, the production of extendedspectrum $\beta$-lactamases (ESBLs) and/or AmpC enzymes in combination with decreased permeability by mutations in outer membrane proteins OmpK35 and OmpK36, as well as over-expression of efflux pumps play roles in the carbapenem resistance [11, 12]. It is noteworthy that CRE infections are closely associated with high mortality because of limited antimicrobial use [13]. To date, fosfomycin, minocycline, tigecycline, colistin, ceftazidime/avibactam and aztreonam/avibactam have been recommended for treatment of infections caused by CRE [14]. However, data on susceptibilities of these antibiotics on CRE are still limited.

Although CRE has been frequently reported in China $[8,15]$, most of the analyzed CRE were collected from the Third-Class A General Hospitals, and the information on CRE isolates collected from specialized hospitals, Children's hospital and level II hospitals was less available. Furthermore, data on non-K. pneumoniae CRE are also less available, owing to the focus of globally disseminated $K$. pneumoniae.

In this study, 97 CRE strains were collected from 17 hospitals, including specialized hospitals, Children's hospital and level II hospitals. The antimicrobial susceptibility, resistant determinants, sequence types (STs), as well as plasmid replicons, were analyzed to investigate the epidemiological characteristics of these CRE.

\section{Materials and methods}

\section{Strain collection}

Totally, 97 CRE were collected from 17 hospitals in Nanjing district, among them, 77 were Klebsiella pneumoniae, 11 Escherichia coli, 4 Citrobacter freundii, 2 Escherichia cloacae, 2 Klebsiella oxytoca and 1 Serratia marcescens. The following hospitals were involved in this investigation: Nanjing Drum Tower Hospital $(n=20)$, Second Affiliated Hospital of Nanjing Medical University $(n=13)$, Nanjing Maternal and Child Health Hospital $(n=2)$, Jiangning Branch of Zhongda Hospital Affiliated to Southeast University $(n=1)$, Nanjing Meishan Hospital $(n=3)$, Nanjing Liuhe Hospital $(n=3)$, Nanjing First Hospital $(n=5)$, Nanjing jinyu Hospital $(n=4)$, Nanjing Brain Hospital $(n=7)$, Nanjing Lishui Hospital $(n=1)$, Nanjing Dachang Hospital $(n=9)$, Nanjing Thoracic Hospital $(n=7)$, Nanjing Children's Hospital $(n=6)$, Nanjing Traditional Chinese Medicine Hospital $(n=4)$, Gaochun People's Hospital $(n=2)$, Nanjing Mingji Hospital $1(n=8)$, and Jiangbei People's Hospital $(n=2)$. All strains were confirmed by VITEK 2.0 or ATB 32E (bioMérieux. Firenze, Italy). The strains were collected from the following samples: sputum $(n=34)$, urine $(n=13)$, secretion $(n=5)$, blood $(n=3)$, bile $(n=1)$, and pus $(n=$ $1)$, others remained unknown.

\section{MIC determination}

In total, 27 antimicrobial agents were used to test the susceptibilities of the CRE. Among them, 25 were tested by micro-broth dilution method, including ertapenem, imipenem, meropenem, cefepime, ceftazidime, cefotaxime, cefuroxime, cefazolin, cefmetazole, piperacillin/tazobactam, amikacin, gentamicin, funantuoyin, trimethoprim and sulphame-thoxazole, aztreonam, piperacillin, ciprofloxacin, levofloxacin, aztreonam/avibactam, ceftazidime/avibactam, tigecycline, and colistin. The susceptibilities toward fosfomycin and minocycline were determined by Kirby-Bauer method. Results were interpreted according to the guideline of CLSI 2019 [16]. Considering the absence of CLSI breakpoints for interpretation of tigecycline and colistin results, the current European Committee on Antimicrobial Susceptibility Testing (EUCAST) (www.eucast.org) guidelines were used. For tigecycline, a cutoff MIC of $\leq 1 \mu \mathrm{g} / \mathrm{ml}$ and $>$ $2 \mu \mathrm{g} / \mathrm{ml}$ was used as the susceptibility and resistance breakpoints, respectively. For colistin, a cutoff MIC of $\leq 2 \mu \mathrm{g} / \mathrm{ml}$ was taken as susceptibility breakpoint. The $E$. coli ATCC25922 was used as quality control. Carbapenem resistance was defined as a MIC of $\geq 2 \mu \mathrm{g} / \mathrm{ml}$ for ertapenem or a MIC of $\geq 4 \mu \mathrm{g} / \mathrm{ml}$ for imipenem or meropenem.

\section{Detection of resistant determinants}

DNA templates were prepared by the boiling method. All the 97 strains were detected by PCR for carbapenemase encoding genes (blaKPC, blaAIM, blaSIM, blaSPM, blaVIM, blaIMP, blaOXA, blaDIM, blaNDM, blaGIM, and blaGES), PMQR (qnrA, qnrB, qnrC, qnrD, $q n r S$, aac (6')-Ib-cr, and qepA), blaESBL encoding genes (blaCTX, blaTEM, blaSHV, blaOXA, blaVEB, and blaPER), 16S-RMTases encoding genes (armA, npmA, 
$r m t A, r m t B, r m t C, r m t D$, and $r m t E)$ and $\mathrm{pAmpC}$ encoding genes (blaEBC, blaMOX, blaACC, bla FOX, blaDHA, and blaCIT) according to the methods described previously [17-21]. The purified PCR products were sent to the Qingke Biotechnology Co., Ltd. (Nanjing, China) for sequencing. Sequences were analyzed by using the Chromas-Pro application and BLAST (www. ncbi.nlm.nih.gov/BLAST), and the subtypes of $\beta$ lactamase genes were confirmed by referring to the Lahey system (www.lahey.org/studies/).

\section{Multi-locus sequence typing}

The STs of CRE were determined by multi-locus sequence typing (MLST). Seven CRKP housekeeping genes, including gapA, infB, $m d h$, pgi, phoE, rpoB, and $\operatorname{ton} B$ were amplified and sequenced according to Yang et al. [22]. The MLST database (www.pasteur.fr/mlst/ Kpneumoniae.html) was used to assign the alleles and STs. For E. coli, the 7 housekeeping genes, including $a d k$, fum $C$, gyrB, icd, $m d h$, purA, and recA were amplified and analyzed according to the protocol available at https://bigsdb.pasteur.fr/ecoli/ecoli.html. The clonal lineages of $E$. cloacae were determined by analyzing the 7 housekeeping genes (dnaA, fusA, gyrB, leuS, pyrG, rplB, and $r р о B$ ) following the scheme developed by MiyoshiAkiyama et al. [23], and the STs were assigned according to the protocols on the MLST website (http://pubmlst. org/ecloacae/). The MLST analysis of $C$. freundii were performed according to the protocol provided on the website (https://pubmlst.org/cfreundii/), with the housekeeping genes $a s p C, \operatorname{clpX}, f a d D, m d h, \operatorname{arc} A, d n a G$ and lys $P$. Additionally, the STs of the $K$. oxytoca was analyzed by assigning the definite 7 housekeeping genes (gapA, $\operatorname{infB}, m d h, p g i$, phoE, rpoB, and tonB) according to the protocol (https://pubmlst.org/koxytoca/).

\section{PCR-based replicon typing}

In order to determine the distribution of plasmid incompatibility groups among the CRE, thirty different plasmid replicons including $\mathrm{HI} 1, \mathrm{HI} 2, \mathrm{I} 1, \mathrm{I} 2, \mathrm{X} 1, \mathrm{X} 2, \mathrm{X} 3, \mathrm{X} 4, \mathrm{~L}$, M, N, FIA, FIB, FIC, FII, FIIs, FIIk, FIB-KN, FIB-KQ, W, $\mathrm{Y}, \mathrm{P} 1, \mathrm{~A} / \mathrm{C}, \mathrm{T}, \mathrm{K}, \mathrm{U}, \mathrm{R}, \mathrm{B} / \mathrm{O}, \mathrm{HIB}-\mathrm{M}$ and FIB-M were determined by using PCR-based replicon typing (PBRT)KIT 2.0 (DIATHEVA, Italy).

\section{Statistical analysis}

SPSS software (20.0) was used to implement statistical analysis. The differences on distribution of resistant determinants/plasmid replicons between bacteria were analyzed by Chi-square test, and the differences were considered to be significant when $p$ value was less than 0.05 ; The differences on distribution of antimicrobial resistance determinants and plasmid replicons among CRE were analyzed by McNemar test. The distribution rates were considered to be the same when $\mathrm{p}$ value was more than 0.05 .

\section{Results \\ MIC determination}

On the whole, none of the $97 \mathrm{CRE}$ strains were susceptible to $\beta$-lactam antibiotics, including imipenem, meropenem, ertapenem, cefepime, ceftazidime, cefotaxime, cefuroxime, cefazolin, piperacillin, ciprofloxacin and levofloxacin. The non-susceptible rates to aztreonam, gentamycin, amikacin were 96.9, 79.4 and $61.9 \%$, respectively. Meanwhile, $86.6 \%$ of the CRE were nonsusceptible to trimethoprim-sulfamethoxazole and 59.8\% to nitrofurantoin. In addition, 31 (32.0\%) strains were non-susceptible towards minocycline and 59 (60.8\%) to fosfomycin. The good side was that most CRE were still susceptible to aztreonam/avibactam and ceftazidime/avibactam, colistin and tigecycline, with susceptibility rates being 96.9, 78.4, 95.9 and 96.9\%, respectively. The relative MIC50 and MIC90 were summarized in Table 1.

\section{Prevalence of resistance determinants}

The prevalence of resistance determinants was shown in Table 2. Among the 97 CRE strains, 92 (94.8\%) CRE carried carbapenemase encoding genes, which were 77 blaKPC-2, 19 blaNDM and 2 blaIMP-4. Ninety-three (95.9\%) CRE were found to co-carry more than three resistance determinants. Remarkably, three E. coli cocarried blaKPC-2 and blaNDM-5. Co-existence of blaIMP-4 and blaKPC-2, and co-occurrence of blaKPC2 and blaNDM-5 were also found in the two K. pneumoniae isolates. An E. cloacae was found to simultaneously carry blaNDM-1 and blaIMP-4.

Analysis of ESBL encoding genes found 73 blaTEM-1, 7 blaCTX-variants, 11 SHV-variants, and 2 blaOXA variants. In detail, 7 blaCTX-variants were composed of blaCTX-M-65 $(n=34)$, blaCTX-M-15 $(n=18)$, blaCTXM-14 $(n=8)$, blaCTX-M-3 $(n=4)$, blaCTX-M-24 $(n=$ 2), blaCTX-M-55 $(n=6)$ and blaCTX-M-45 $(n=1)$. Similarly, 11 SHV-variants were divided into blaSHV-11 $(n=23)$, blaSHV-12 $(n=9)$, blaSHV-28 $(n=8)$, blaSHV$182(n=8)$, blaSHV-13 $(n=4)$, blaSHV-1 $(n=2)$, blaSHV-67 $(n=1)$, blaSHV-36 $(n=1)$, blaSHV-172 $(n=$ $1)$, blaSHV-15 $(n=1)$ and blaSHV-190 $(n=1)$. Two bla OXA variants were blaOXA- $1(n=14)$ and blaOXA$10(n=2)$. Moreover, pAmpCs analysis showed prevalence of blaDHA-1 $(n=10)$, followed by 5 blaCMY variants, including blaCMY-2 $(n=4)$, blaCMY-65 $(n=2)$, blaCMY-77 $(n=2)$, blaCMY-34 $(n=1)$, and blaCMY-42 $(n=1)$; blaACT-16 $(n=1)$, blaSRT-1 $(n=2)$, blaOXY-2$2(n=1)$ and blaSFO-1 $(n=1)$ were also detected. For PMQRs, there were 68 oqxAB, 62 qnr-variants, 22 $a a c\left(6^{\prime}\right)-I b-c r$ and $1 q e p A$. The variants of $q n r$ were as follows: qnrA1 $(n=2)$, qnrB4 $(n=10)$, qnrB7 $(n=1)$, 
Table 1 The $\mathrm{MIC}_{50}$ and $\mathrm{MIC}_{90}$ of the carbapenem resistant enterobacteraceae isolates

\begin{tabular}{|c|c|c|c|}
\hline Antibiotics & $\mathrm{MIC}_{50}$ & $\mathrm{MIC}_{90}$ & Range $(\mu \mathrm{g} / \mathrm{ml})$ \\
\hline ertapenem & $>32$ & $>32$ & $0.25-32$ \\
\hline imipenem & $>16$ & $>16$ & $0.125-16$ \\
\hline meropenem & $>16$ & $>16$ & $0.125-16$ \\
\hline cefepime & $>32$ & $>32$ & $0.25-32$ \\
\hline ceftazidime & $>32$ & $>32$ & $0.25-32$ \\
\hline cefotaxime & $>32$ & $>32$ & $0.25-32$ \\
\hline cefuroxim & $>64$ & $>64$ & $0.5-64$ \\
\hline cefazolin & $>32$ & $>32$ & $0.25-32$ \\
\hline cefmetazole & $>64$ & $>64$ & $0.5-64$ \\
\hline piperacillin/tazobactam & $>256 / 4$ & $>256 / 4$ & $2 / 4-245 / 4$ \\
\hline amikacin & $>128$ & $>128$ & $1-128$ \\
\hline gentamicin & $>128$ & $>128$ & $1-128$ \\
\hline funantuoyin & $>128$ & $>128$ & $1-128$ \\
\hline trimethoprim and sulphame-thoxazole & 2 & $>32$ & $0.25 / 4.75-32 / 608$ \\
\hline aztreonam & $>128$ & $>128$ & $1-128$ \\
\hline piperacillin & $>256$ & $>256$ & $2-256$ \\
\hline ciprofloxacin & $>8$ & $>8$ & $0.06-8$ \\
\hline levofloxacin & $>16$ & $>16$ & $0.125-8$ \\
\hline aztreonam/avibactam & 0.5 & 2 & $0.25-32$ \\
\hline ceftazidime/avibactam & 0.25 & 1 & $0.25-32$ \\
\hline tigecycline & 0.5 & 1 & $0.125-16$ \\
\hline colistin & 0.5 & 0.5 & $0.125-16$ \\
\hline
\end{tabular}

qnrB18 $(n=2)$, qnrS1 $(n=34)$, and qnrS2 $(n=3)$. Finally, 16S-RMTase encoding genes $r m t B(n=46)$ and $\operatorname{arm} A$ $(n=3)$, fosfomycin resistant genes fos $A(n=74)$, fos $A 3$ $(n=36)$ and fosA5 $(n=5)$ were also found.

For the 77 CRKP strains, 70 blaKPC-2, 5 blaNDM and 6 blaCTX-M variants were identified with blaCTX-M$65(n=32)$, being the predominant resistance determinant, followed by blaCTX-M-15 $(n=16)$ and blaCTX-M$14(n=5)$. Ten blaSHV variants containing blaSHV-11 $(n=18)$, blaSHV-28 $(n=8)$, blaSHV-12 $(n=7)$, blaSHV$182(n=7)$, blaSHV-13 $(n=4)$, blaSHV-1 $(n=1)$, blaSHV-15 $(n=1)$, blaSHV-36 $(n=1)$, blaSHV-67 $(n=$ $1)$, and blaSHV-172 $(n=1)$ were found. The other main genes identified included blaTEM-1B $(n=60)$, blaDHA$1(n=7)$, blaOXA-1 $(n=8), r m t B(n=41)$, aac $\left(6^{\prime}\right)-I b-c r$ $(n=14), o q x A B(n=62), q n r S 1(n=27)$, and qnrB4 $(n=$ 7). Additionally, 71 (92.2\%) out of 77 CRKP strains carried fos $A$ genes, among which, 31 (40.2\%) co-carried fos $A$ and fos $A 3,5$ fos $A 5$ were also found. Among the 11 CREC, there were 3 blaKPC-2 and 11 blaNDM. Additionally, blaKPC-2 was identified in $2 \mathrm{C}$. freundii, $1 \mathrm{~S}$. marcescens and $1 E$. cloacae, blaNDM was found in $1 C$. freundii, $1 \mathrm{~K}$. oxytoca and $1 \mathrm{E}$. cloacae.

Overall, K. pneumoniae strains carried much more blaKPC-2, blaCTX-M-65, blaTEM-1B, rmtB, fos $A$, and
oqxAB genes compared with other Enterobacteriaceae. In particular, blaKPC-2, blaDHA-1, fos $A$, oq $x A B$ and $r m t B$ were higher in $K$. pneumoniae than those in $E$. coli. Whereas, more blaNDM and $q n r$ were distribued among $E$. coli than those in $K$. pneumoniae. As well, more blaNDM, blaOXA, pAmpCs, acc(6')-Ib-cr and $q n r$ in other Enterobacteriaceae were prevalent than those in $K$. pneumoniae strains (Table 2). The distribution of resistance determinants among the remaining five nocarbapenemase-producing CRE strains were summarized in Table 3.

\section{CRE sequence types}

Among the 77 CRKP isolates, 13 STs were identified, with ST11 $(n=54)$ being the dominant one, followed by ST15 $(n=7)$ and ST268 $(n=5)$. The other STs included ST942 $(n=2), \operatorname{ST} 48(n=1), \operatorname{ST} 290(n=1), \operatorname{ST} 1779(n=$ $1), \operatorname{ST} 23(n=1), \operatorname{ST} 65(n=1), \operatorname{ST} 86(n=1), \operatorname{ST} 577(n=$ $1)$ ST17 $(n=1)$, and ST1 $(n=1)$. There were 7 STs in 8 CREC, which were ST410 $(n=2)$, ST3489 $(n=1)$, ST156 $(n=1), \operatorname{ST} 683(n=1), \operatorname{ST} 297(n=1), \operatorname{ST} 167(n=1)$, and ST361 $(n=1)$. In addition, $3 E$. coli strains could not be assigned to specific STs. The STs for $4 \mathrm{C}$. freundii strains were ST116 $(n=2), \operatorname{ST} 248(n=1)$ and ST36 $(n=1)$. The STs for $2 K$. oxytoca strains were ST105 and 
Table 2 The distribution of resistance determinants among the carbapenem resistance enterobacteraceae isolates

\begin{tabular}{|c|c|c|c|c|c|c|c|c|c|}
\hline $\begin{array}{l}\text { Resistant } \\
\text { determinants }\end{array}$ & $\begin{array}{l}\text { K. } \\
\text { pneumoniae } \\
(n=77)\end{array}$ & $\begin{array}{l}\text { K. oxytoca } \\
(n=2)\end{array}$ & $\begin{array}{l}\text { E. coli } \\
(n=11)\end{array}$ & $\begin{array}{l}\text { S. } \\
\text { marcescens } \\
(n=1)\end{array}$ & $\begin{array}{l}\text { C. freundii } \\
(n=4)\end{array}$ & $\begin{array}{l}\text { E. cloacae } \\
(n=2)\end{array}$ & $\begin{array}{l}\text { Enterobacter } \\
(n=20)\end{array}$ & $\begin{array}{l}P \text { value ( } K . \\
\text { pneumoniae Vs } E . \\
\text { coli) }\end{array}$ & $\begin{array}{l}P \text { value (K. pneumoniae } \\
\text { Vs Enterobacter) }\end{array}$ \\
\hline $\begin{array}{l}\text { blaKPC-2 } \\
(n=77)\end{array}$ & 70 & 0 & 3 & 1 & 2 & 1 & 7 & 0.000 & 0.000 \\
\hline $\begin{array}{l}\text { blaNDM }(n= \\
19)\end{array}$ & 5 & 1 & 11 & 0 & 1 & 1 & 14 & 0.000 & 0.000 \\
\hline $\begin{array}{l}\text { blaCTX-M } \\
(n=74)\end{array}$ & 59 & 0 & 10 & 1 & 4 & 0 & 15 & 0.493 & 0.879 \\
\hline $\begin{array}{l}\text { blacTX-M-15 } \\
(n=18)\end{array}$ & 16 & 0 & 2 & 0 & 0 & 0 & 2 & 1.000 & 0.434 \\
\hline $\begin{array}{l}\text { blacTX-M-65 } \\
(n=34)\end{array}$ & 32 & 0 & 1 & 0 & 1 & 0 & 2 & 0.081 & 0.018 \\
\hline $\begin{array}{l}\text { blaSHV }(n= \\
\text { 59) }\end{array}$ & 49 & 0 & 5 & 0 & 3 & 2 & 10 & 0.247 & 0.266 \\
\hline $\begin{array}{l}\text { blaSHV- } \\
11(n=23)\end{array}$ & 18 & 0 & 3 & 0 & 1 & 1 & 5 & 1.000 & 0.879 \\
\hline $\begin{array}{l}\text { blaTEM }(n= \\
73)\end{array}$ & 60 & 1 & 8 & 0 & 2 & 2 & 13 & 1.000 & 0.233 \\
\hline $\begin{array}{l}\text { blaTEM- } \\
1 \mathrm{~B}(n=71)\end{array}$ & 60 & 1 & 7 & 0 & 1 & 2 & 11 & 0.508 & 0.039 \\
\hline $\begin{array}{l}\text { blaOXA }(n= \\
16)\end{array}$ & 8 & 1 & 4 & 0 & 3 & 0 & 8 & 0.060 & 0.001 \\
\hline $\begin{array}{l}\text { blaOXA-1 } \\
(n=14)\end{array}$ & 8 & 1 & 4 & 0 & 1 & 0 & 6 & 0.060 & 0.026 \\
\hline $\begin{array}{l}\text { pAmpCs } \\
(n=22)\end{array}$ & 9 & 3 & 3 & 1 & 5 & 1 & 13 & 0.348 & 0.000 \\
\hline $\begin{array}{l}\text { blaDHA-1 } \\
(n=10)\end{array}$ & 7 & 1 & 0 & 0 & 2 & 0 & 3 & 0.000 & 0.718 \\
\hline $\begin{array}{l}\operatorname{og} \times A B(n= \\
68)\end{array}$ & 62 & 0 & 2 & 1 & 2 & 1 & 6 & 0.000 & 0.000 \\
\hline $\operatorname{Qnr}(n=62)$ & 37 & 3 & 16 & 1 & 5 & 0 & 25 & 0.021 & 0.000 \\
\hline $\begin{array}{l}\text { qnrB4 }(n= \\
10)\end{array}$ & 7 & 1 & 0 & 0 & 2 & 0 & 3 & 0.339 & 0.718 \\
\hline $\begin{array}{l}\text { anrS1 }(n= \\
34)\end{array}$ & 27 & 1 & 4 & 1 & 1 & 0 & 7 & 0.309 & 0.996 \\
\hline $\begin{array}{l}a a c\left(6^{\prime}\right) \mid b-c r \\
(n=22)\end{array}$ & 14 & 0 & 4 & 0 & 3 & 1 & 8 & 1.000 & 0.038 \\
\hline$r m t B(n=46)$ & 41 & 0 & 2 & 0 & 2 & 1 & 5 & 0.001 & 0.024 \\
\hline fOSA $(n=74)$ & 71 & 0 & 0 & 0 & 1 & 2 & 3 & 0.000 & 0.000 \\
\hline fosA3 $(n=36)$ & 31 & 1 & 3 & 0 & 1 & 0 & 5 & 0.065 & 0.208 \\
\hline
\end{tabular}

Table 3 The sequence types and distribution of resistance determinants of CRE strains without producing carbapenemase

\begin{tabular}{|c|c|c|c|c|c|c|c|c|c|}
\hline code & Strain & STs & CTX-M & SHV & OXA & pAmpC & PQMRs & 16S-RMTase & FRG \\
\hline 6 & K. pneumoniae & ST11 & blacTX-M-15 & blaSHV-28 & blaOXA-1 & & $o q \times A B, a n r S 1, \operatorname{acc}\left(6^{\prime}\right)-l b-c r$ & & \\
\hline 54 & K. pneumoniae & ST15 & blactX-M-15 & & blaOXA-1 & & $a c c\left(6^{\prime}\right)-1 b-c r$ & & \\
\hline 64 & C. freundii & ST248 & blacTX-M-65, blaCTX-M-3 & blaSHV-11 & & blaCMY-65 & oqxAB, $\operatorname{acc}\left(6^{\prime}\right)-1 b-c r$ & $\operatorname{arm} A, r m t B$ & \\
\hline 65 & K. oxytoca & ST105 & & & & blaCMY-77 blaDHA-1 & qnrB18, qnrB4 & & fOSA \\
\hline 933 & K. pneumoniae & ST290 & blaCTX-M-15 & oqXAB & & blaDHA-1 & qnrB4 & & \\
\hline
\end{tabular}


ST36, respectively, and the two E. cloacae were assigned to ST418 and ST723.

\section{Plasmid replicons}

The distribution of plasmid replicons was shown in Table 4. Among the 97 CRE strains, 10 strains only carried one plasmid replicon, 82 strains carried two or more plasmid replicons, whereas, 5 strains seemed not to contain any type of plasmids. In total, 15 types of plasmid replicons were detected among the $97 \mathrm{CRE}$, including IncR $(n=64)$, IncFII $(n=63)$, IncFIB $(n=45)$, IncX3 $(n=16)$, $\operatorname{IncFII}_{\mathrm{K}}(n=13)$, IncFIA $(n=10)$, IncFIC $(\mathrm{n}=2), \operatorname{IncH} 11(n=8), \operatorname{IncH} 12(\mathrm{n}=4), \operatorname{IncA} / \mathrm{C}(n=5)$, $\operatorname{IncX1}(n=2), \operatorname{IncN}(n=1), \operatorname{IncP1}(n=1), \operatorname{IncB} / \mathrm{O}(n=1)$ and IncI1 $(n=1)$. Of these replicon-types, the combination of IncFII and IncR was the most common and mainly found in KPC-2 producers $(n=54)$. Nine types of replicons were detected in CRKP, of which IncR $(n=59)$ dominated, followed by IncFII $(n=55)$, IncFIB $(n=34)$ and $\operatorname{IncFII}_{\mathrm{K}}(n=13)$. Regarding CREC, 13 types of plasmid replicons were identified, with $\operatorname{IncX} 3(n=11)$ and IncFIB $(n=8)$ being the most prevalent types.

Compared with other Enterobacteriaceae species, $K$. pneumoniae had obviously higher distribution of IncFII, IncR and $\operatorname{IncFII}_{\mathrm{K}}(p<0.05)$. In contrast, the distribution of IncX3 was lower in the $K$. pneumoniae strains than those among other Enterobacteriaceae species $(p<0.05)$.

No significant differences were shown on the distributions between some main resistance determinants and specific plasmid replicons among the CRE (Table 5), such as blaNDM, blaCTX-M-15, blaDHA-1, qnrB4 and $a a c\left(6^{\prime}\right)-I b-c r$ with IncX3, IncFIIk, and IncFIA, blaCTX$\mathrm{M}$ and $o q x A B$ with IncFII and IncR, blaCTX-M-65 with IncFIB, pAmpCs with IncX3, rmtB and IncFIB, fos $A$ with IncR, as well as fos $A 3$ with IncFIB (Table 5). Furthermore, the consistency on most of these distributions could also be observed among the CRKP (Table 6), and more detailed distribution correlations were further displayed between the following resistance determinants and plasmid replicons: blaCTX-M-15 with $\mathrm{IncFII}_{\mathrm{K}}$, $q n r$ and qnrS1 with IncFIB, $r m t B$ with IncFIB, fos $A 3$ with IncFIB, $a a c\left(6^{\prime}\right)-I b-c r$ with IncFII $_{K}$.

\section{Discussion}

In this study, we provided data on the antimicrobial resistance profiles, STs, and plasmid replicons profiles of CRE isolates collected from specialized hospitals, Children's hospital and level II hospitals in Nanjing district, Southeast China.

Most of our CRE strains displayed high resistance against the commonly used antimicrobial agents, which was consistent with previous report, providing evidence that the CRE strains are usually resistant to many other classes of antibiotics in clinical practice [24]. Fortunately, most of the CRE strains were still susceptible towards tigecycline, colistin, ceftazidime/avibactam, and aztreonam/avibactam. Resistance to aztreonam/avibactam and ceftazidime/avibactam have appeared in our study, which may be attributed to mutations in the carbepenamase KPC and NDM [25], suggesting a rapid resistance development of $K$. pneumoniae. None carbepenemase encoding genes were detected among the 5 CRE strains in our study, where the production of ESBLs variants and/or AmpC enzymes in combination of overexpressed efflux pumps or the decreased permeability might contribute to the carbapenem resistance.

The high prevalence of blaKPC-2 and many other resistance related genes, such as blaESBLs, PMQRs, as well as 16S-RMTase was consistent with our previous report [8], indicating the frequent co-existence or coevolution of antibiotic resistance genes, which might lead to the emergence of untreatable $K$. pneumoniae infections. Among them, the quite high prevalence of blaESBL among the blaKPC-2 producing CRE strains in our study represented crippling and urgent threats to public health, because multiple copies of blaCTX-M and

Table 4 The distribution of major plasmid replicons among carbapenem-resistant Enterobacteriaceae

\begin{tabular}{|c|c|c|c|c|c|c|c|c|}
\hline $\begin{array}{l}\text { Plasmid } \\
\text { replicons }\end{array}$ & $\begin{array}{l}\text { K. pneumoniae } \\
(n=77)\end{array}$ & $\begin{array}{l}\text { K. oxytoca } \\
(n=2)\end{array}$ & $\begin{array}{l}\text { E. coli } \\
(n=11)\end{array}$ & $\begin{array}{l}\text { S. marcescens } \\
(n=1)\end{array}$ & $\begin{array}{l}\text { C. freundii } \\
(n=4)\end{array}$ & $\begin{array}{l}\text { E.cloacae } \\
(n=2)\end{array}$ & $\begin{array}{l}P \text { value ( } K \text {. } \\
\text { pneumoniae Vs E. coli) }\end{array}$ & $\begin{array}{l}P \text { value (K. pneumoniae Vs } \\
\text { Enterobacter) }\end{array}$ \\
\hline $\begin{array}{l}\operatorname{lncR}(n= \\
64)\end{array}$ & 59 & 2 & 1 & 1 & 0 & 1 & 0.000 & 0.000 \\
\hline $\begin{array}{l}\operatorname{lncFII}(n= \\
63)\end{array}$ & 55 & 0 & 6 & 0 & 1 & 1 & 0.256 & 0.009 \\
\hline $\begin{array}{l}\ln C F I B(n= \\
45)\end{array}$ & 34 & 1 & 8 & 0 & 0 & 2 & 0.147 & 0.386 \\
\hline $\begin{array}{l}\operatorname{lnc} X 3(n= \\
16)\end{array}$ & 2 & 1 & 11 & 0 & 1 & 1 & 0.000 & 0.000 \\
\hline $\begin{array}{l}\operatorname{lncFI} \|_{K}(n= \\
13)\end{array}$ & 13 & 0 & 0 & 0 & 0 & 0 & 0.052 & 0.011 \\
\hline $\begin{array}{l}\text { IncFIA }(n= \\
10)\end{array}$ & 7 & 0 & 3 & 0 & 0 & 0 & 0.204 & 0.718 \\
\hline
\end{tabular}


Table 5 The differences on the distribution of plasmid replicons and resistant determinants among the carbapenem-resistant Enterobacteriaceae

\begin{tabular}{|c|c|c|c|c|c|c|}
\hline \multirow{2}{*}{$\begin{array}{l}\text { Resistance } \\
\text { determinants }\end{array}$} & \multicolumn{6}{|c|}{ Plasmid replicons } \\
\hline & IncFII $(n=63)$ & $\operatorname{lncR}(n=64)$ & $\operatorname{lncFIB}(n=45)$ & $\operatorname{lncX3}(n=16)$ & $\operatorname{lncF} \|_{K}(n=13)$ & IncFIA $(n=10)$ \\
\hline blaKPC-2 $(n=77)$ & 0.001 & 0.005 & 0.000 & 0.000 & 0.000 & 0.000 \\
\hline blaNDM $(n=19)$ & 0.000 & 0.000 & 0.000 & 0.754 & 1.000 & 0.503 \\
\hline blaCTX-M ( $n=74)$ & 0.229 & 0.440 & 0.002 & 0.000 & 0.000 & 0.000 \\
\hline blaCTX-M-15 $(n=18)$ & 0.000 & 0.000 & 0.000 & 0.701 & 0.210 & 0.093 \\
\hline blaCTX-M-65 $(n=34)$ & 0.000 & 0.000 & 0.124 & 0.026 & 0.002 & 0.001 \\
\hline blaSHV $(n=59)$ & 0.665 & 0.568 & 0.093 & 0.000 & 0.000 & 0.000 \\
\hline blaSHV-11 ( $(n=23)$ & 0.000 & 0.000 & 0.002 & 0.487 & 0.087 & 0.019 \\
\hline blaTEM $(n=73)$ & 0.087 & 0.121 & 0.000 & 0.000 & 0.000 & 0.000 \\
\hline blaTEM-1B $(n=71)$ & 0.185 & 0.281 & 0.000 & 0.000 & 0.000 & 0.000 \\
\hline blaOXA $(\underline{n}=16)$ & 0.139 & 0.000 & 0.000 & 0.824 & 0.000 & 0.839 \\
\hline blaOXA-1 $(n=14)$ & 0.000 & 0.000 & 0.000 & 0.481 & 1.000 & 1.000 \\
\hline pAmpCs $(n=22)$ & 0.000 & 0.000 & 0.000 & 0.122 & 0.043 & 0.008 \\
\hline blaDHA-1 $(n=10)$ & 0.000 & 0.000 & 0.000 & 0.503 & 1.000 & 0.815 \\
\hline $\operatorname{oq} \times A B(n=68)$ & 0.720 & 1.000 & 0.009 & 0.000 & 0.000 & 0.000 \\
\hline $\operatorname{qnr}(n=62)$ & 0.235 & 0.275 & 0.262 & 0.000 & 0.000 & 0.000 \\
\hline qnrB4 $(n=10)$ & 0.000 & 0.000 & 0.000 & 0.701 & 1.000 & 0.648 \\
\hline qnrs1 $(n=34)$ & 0.000 & 0.000 & 0.073 & 0.009 & 0.000 & 0.000 \\
\hline $\operatorname{aac}\left(6^{\prime}\right) \mid b-c r(n=22)$ & 0.000 & 0.000 & 0.000 & 0.557 & 0.230 & 0.087 \\
\hline$r m t B(n=46)$ & 0.004 & 0.014 & 0.659 & 0.000 & 0.000 & 0.000 \\
\hline fosA $(n=74)$ & 0.017 & 0.152 & 0.000 & 0.000 & 0.000 & 0.000 \\
\hline fosA3 $(n=36)$ & 0.000 & 0.000 & 0.322 & 0.002 & 0.001 & 0.000 \\
\hline
\end{tabular}

Table 6 The differences on the distribution of plasmid replicons and resistant determinants among the carbapenem resistant $K$. pneumoniae

\begin{tabular}{|c|c|c|c|c|c|}
\hline \multirow{2}{*}{$\begin{array}{l}\text { Resistance } \\
\text { determinants }\end{array}$} & \multicolumn{5}{|c|}{ Plasmid replicons } \\
\hline & FII $(n=55)$ & $\mathrm{R}(n=59)$ & $\mathrm{FIB}(n=34)$ & $\mathrm{FII}_{\mathrm{K}}(n=13)$ & $\mathrm{FIA}(n=7)$ \\
\hline blaKPC-2 $(n=70)$ & 0.001 & 0.007 & 0.000 & 0.000 & 0.000 \\
\hline blacTX-M $(n=59)$ & 0.572 & 1.000 & 0.000 & 0.000 & 0.000 \\
\hline blaCTX-M-15 $(n=16)$ & 0.000 & 0.000 & 0.001 & 0.629 & 0.049 \\
\hline blaCTX-M-65 $(n=32)$ & 0.000 & 0.000 & 0.890 & 0.005 & 0.000 \\
\hline blaSHV $(n=49)$ & 0.405 & 1.000 & 0.000 & 0.000 & 0.000 \\
\hline blaSHV-11 $(n=18)$ & 0.510 & 0.000 & 0.011 & 0.424 & 0.027 \\
\hline blaTEM $(n=60)$ & 0.405 & 1.000 & 0.000 & 0.000 & 0.000 \\
\hline $\operatorname{oq} \times A B(n=62)$ & 0.839 & 0.845 & 0.001 & 0.000 & 0.000 \\
\hline Qnr $(n=37)$ & 0.025 & 0.002 & 0.690 & 0.000 & 0.000 \\
\hline anrs1 $(n=27)$ & 0.000 & 0.000 & 0.210 & 0.009 & 0.000 \\
\hline$a a c\left(6^{\prime}\right) \mid b-c r(n=14)$ & 0.000 & 0.000 & 0.167 & 1.000 & 0.000 \\
\hline$r m t B(n=41)$ & 0.003 & 0.000 & 0.324 & 0.000 & 0.000 \\
\hline fOSA $(n=71)$ & 0.000 & 0.004 & 0.000 & 0.000 & 0.000 \\
\hline fosA3 $(n=31)$ & 0.000 & 0.000 & 0.742 & 0.008 & 0.000 \\
\hline
\end{tabular}


blaKPC within plasmids could be integrated and disseminated into chromosome [26]. In that case, the spread of such strains would be horizontally and vertically accelerated within hospitals. To date, the co-occurrence of blaCTX-M-65 and blaKPC-2 in our study has been previously reported in $K$. pneumoniae [27]. Notably, we found quite high prevalence of fosA in blaKPC-2 producing $K$. pneumoniae. fos $A$ has been reported to be chromosomally encoded by clinically relevant Gram-negative species and contributes to intrinsic fosfomycin resistance [28]. However, some strains carrying fos $A$ in our study displayed susceptibility to fosfomycin, which needs to be further investigated. Furthermore, the wide distribution of fos $A$ and fos $A 3$, as well as the emergence of fos $A 5$, indicated that fosfomycin should be cautiously used for treating the CRKP infections, since the combination of plasmid-borne fos $A 3$ and blaKPC-2 could accelerate the spread of antibiotic resistance [29]. Another interesting point is that, blaCTX-M-45 used to be identified by an algorithm [30]. However, this is the first time that we found this gene in a clinical C. freundii isolate. Among these SHV encoding genes, the blaSHV-13 was discovered in $K$. pneumoniae isolates in Amsterdam [31], whereas, the blaSHV-67, blaSHV-172, blaSHV-182, and blaSHV-190 are brand new, and have never been reported previously. OXA-10-type class D $\beta$-lactamases (previously shown to be weak carbapenemases) was firstly identified in our clinical C. freundii [32]. Moreover, CMY-34 was identified in C. freundii isolate in Danish army recruits, and was also found in our study [33]. To the best of our knowledge, the two blaCMY-65 producing $C$. freundii strains, one blaCMY-77 producing $K$. pneumoniae isolate, one blaCMY-77 producing $K$. oxytoca and a blaACT-16 producing E. cloacae found in our study have not been reported previously. For the first time, we found the co-occurrence of blaDHA-1 and blaCMY-65 in C. freundii, and the co-occurrence of blaCMY-77 and blaDHA-1 in K. oxytoca.

The expansion of ST11 for the KPC-2 producing $K$. pneumoniae was in accordance with previous report indicating that the ST11 is the dominating epidemic clone among CRKP [15]. Albeit the KPC-2 producing E. coli ST410 has been reported, as far as we know, the blaNDM-5 and blaKPC-2 co-carrying E. coli ST167, blaNDM-5 carrying $K$. oxytoca ST36, as well as blaIMP-4 and blaNDM-1 co-carrying E. cloacae ST418 identified in our study have not been reported previously. Additionally, the blaKPC-2 has been identified in C. freundii and E. cloacae, and blaNDM-1 in C. freundii. This is the first time that we identified blaKPC-2 in C. freundii ST116 and E. cloacae ST723, and blaNDM-1 in C. freundii ST36.

Plasmids are extra-chromosomal DNA elements representing major reservoirs for horizontal transmission of antibiotic resistance among bacteria [34]. To date, multiple plasmids have been found to be the vesicles for spread of carbapenemase, ESBLs, and PMQRs [34]. The high prevalence of IncFII, IncR and IncFIB plasmid replicons in our study alert us on the urgency of implementing antimicrobial resistance surveillance, since IncFII-type plasmids are highly distributed vesicles for resistant determinants in $\mathrm{En}$ terobacteriaceae [35]; Moreover, IncR plasmid is an important reservoir of multidrug resistance in Enterobacteriaceae strains, because the conserved IncR backbones include the multidrug resistant (MDR) regions [36]; and IncFIB plasmids were reported to be associated with majority of the antimicrobial resistance genes [37]. Similarly, some of the distribution correlations between various plasmid replicons and multiple resistant genes found in our study are consistent with the previous reports [38-40]. Among them, IncX3 plasmid is a narrow-spectrum plasmid widely distributed in E. coli from wildlife in Europe [38], which has also been found to be predominantly associated with fluoroquinolone resistance genes and $\beta$-lactam resistance genes. This is the similar situation in our CRE strains, where IncX3 was also found to be associated with blaNDM and qnrB4. However, the situation in $K$. pneumoniae is still uncertain, because few IncX3 plasmid replicons were found. In addition, plasmids encoding carbapenemases have been demonstrated to play a core role in the rapid spread of CRE [39]. In our study, the distribution correlations between blaNDM and plasmid replicons IncX3, IncFIA and IncFII $_{K}$ also confirmed the proposal, since the spread of blaNDM is involved in diverse and heterogeneous plasmids [40]. As we know, IncX3 plasmid has been an important vehicle with high mobility in worldwide dissemination of blaNDM [41], and IncFIA-type conjugative plasmid encoding blaNDM-1 has been involved in the outbreak caused by K. pneumoniae strains in Tunisia [11]. The IncN and IncH1 plasmids in our study are less common, this is in accordance with the previous study, which revealed that $\mathrm{IncN}$ and $\mathrm{IncH} 1$ plasmids are host-specific, and are more predominant in livestock and horses in Denmark, respectively [42]. However, the appearance of such plasmid replicons in our study may suggest the spread of plasmids among human and farm livestock.

Five isolates lacked any plasmid type in our study, which may be due to the limitations in the PBRT protocol [43]. It's however noteworthy that the co-existence of multiple plasmid replicons in our CRE strains is consistent with previous study [39], which may result from a conserved backbone responsible for regulation and mating pair stabilization [44].

\section{Conclusion}

In addition to wide distribution of CRE isolates in Nanjing district, Southeast China, the high-level carriage of carbapenemases, ESBLs, pAmpCs, 16S-RMTase, PMQRs and major plasmid replicons in our study might be a 
potential challenge regarding the transmissible capability. Moreover, the expansion of ST11 blaKPC-2 carrying $K$. pneumoniae, the new emergence of blaNDM-5 carrying $K$. oxytoca ST36, as well as blaIMP-4 and blaNDM1 co-carrying E. cloacae ST418 are worrying, efficient and sustained control measures are urgently required.

\section{Abbreviations}

16S-RMTases: 165 rRNA methyltransferase; CRE: Carbapenem-resistant Enterobacteriaceae; CREC: Carbapenem resistant Escherichia coli; CRKP: Carbapenem resistance Klebsiella pneumoniae; ESBLs: Extendedspectrum $\beta$-lactamases; KPC: Klebsiella pneumoniae carbapenemase: MIC: Minimum inhibitory concentration; MLST: Multi-locus sequence typing; NDM: New Delhi metallo-ß-lactamase; PBRT: PCR-based replicon typing; PCR: Polymerase Chain Reaction; PMQRs: Plasmid mediated quinolone resistance genes; STs: Sequence types

\section{Acknowledgments}

We are very grateful to the WHO antimicrobial group, National Food Institute, Technic University of Denmark for providing the positive control strains for detecting resistant determinants and plasmid replicons.

\section{Disclaimer}

The views expressed in this article are those of the authors and do not necessarily reflect the official policy or position of the Department of Laboratory Medicine, Nanjing Drum Tower Hospital, the affiliated Hospital of Nanjing University Medical School, and the Clinical Research Center, the second hospital of Nanjing, Nanjing University of Chinese Medicine, Nanjing, 210,003, China.

\section{Authors' contributions}

ZH performed the detection of resistant determinants and plasmid replicons, as well as multi-locus sequence typing; ZK implemented strains collection and antimicrobial susceptibility testing; $\mathrm{CW}, \mathrm{CJH}$ and $\mathrm{ZJ}$ interpreted the data regarding the resistant determinants and plasmid replicons. LC, CL and ZWQ analyzed the results of multi-locus sequence typing and $\mathrm{CXL}$ and $\mathrm{SH}$ designed the work and was a major contributor in writing the manuscript. All authors read and approved the final manuscript.

\section{Funding}

This work was supported by the Nanjing Medical Science and technique Development Foundation (Grant no: QRX17059), the Youth Fund of Jiangsu Province (Grant no. BK20140099) and National Natural Science Foundation of China (81902124).

\section{Availability of data and materials}

The datasets used and/or analyzed during the current study are available from the corresponding author on reasonable request.

\section{Ethics approval and consent to participate}

Not applicable.

\section{Consent for publication}

Not applicable.

\section{Competing interests}

The authors declare that they have no competing interests.

\section{Author details}

'Department of Laboratory Medicine, Nanjing Drum Tower Hospital, the affiliated Hospital of Nanjing University Medical School, Zhongshan Road 321, Gulou District, Nanjing, Jiangsu, People's Republic of China. ${ }^{2}$ Clinical Research Center, the second hospital of Nanjing, Nanjing University of Chinese Medicine, Nanjing 210003, China.
Received: 20 August 2019 Accepted: 23 December 2019

Published online: 13 January 2020

\section{References}

1. Logan LK, Weinstein RA. The Epidemiology of Carbapenem-Resistant Enterobacteriaceae: The Impact and Evolution of a Global Menace. J Infect Dis. 2017;215(suppl_1):S28-s36.

2. Cerqueira GC, Earl AM, Ernst CM, Grad YH. Multi-institute analysis of carbapenem resistance reveals remarkable diversity, unexplained mechanisms, and limited clonal outbreaks 2017;114(5):1135-1140.

3. Zhang $Y$, Wang $Q$, Yin $Y$, Chen $H$, Jin L, Gu B, et al. Epidemiology of Carbapenem-Resistant Enterobacteriaceae Infections: Report from the China CRE Network. Antimicrob Agents Chemother. 2018;62(2). https://doi.org/10. 1128/AAC.01882-17

4. Grundmann $\mathrm{H}$, Glasner $\mathrm{C}$, Albiger $\mathrm{B}$, Aanensen DM, Tomlinson $\mathrm{CT}$, Andrasevic AT, et al. Occurrence of carbapenemase-producing Klebsiella pneumoniae and Escherichia coli in the European survey of carbapenemase-producing Enterobacteriaceae (EUSCAPE): a prospective, multinational study. Lancet Infect Dis. 2017;17(2):153-63.

5. Patel G, Bonomo RA. "stormy waters ahead": global emergence of carbapenemases. Front Microbiol. 2013;4:48

6. Munoz-Price LS, Poirel L, Bonomo RA, Schwaber MJ, Daikos GL, Cormican M, et al. Clinical epidemiology of the global expansion of Klebsiella pneumoniae carbapenemases. Lancet Infect Dis. 2013;13(9):785-96.

7. Khan AU, Maryam L, Zarrilli R. Structure, Genetics and Worldwide Spread of New Delhi Metallo-beta-lactamase (NDM): a threat to public health. BMC Microbiol. 2017;17(1):101

8. Cheng L, Cao XL, Zhang ZF, Ning MZ, Xu XJ, Zhou W, et al. Clonal dissemination of KPC-2 producing Klebsiella pneumoniae ST11 clone with high prevalence of oqxAB and rmtB in a tertiary hospital in China: results from a 3-year period. Ann Clin Microbiol Antimicrob. 2016;15:1.

9. Guducuoglu H, Gursoy NC, Yakupogullari Y, Parlak M, Karasin G, Sunnetcioglu M, et al. Hospital Outbreak of a Colistin-Resistant, NDM-1- and OXA-48-Producing Klebsiella pneumoniae: High Mortality from Pandrug Resistance. Microb Drug Resist. 2018;24(7):966-72.

10. Porse A, Schonning K, Munck C, Sommer MO. Survival and evolution of a large multidrug resistance plasmid in new clinical bacterial hosts. Mol Biol Evol. 2016;33(11):2860-73.

11. Hamzaoui Z, Ocampo-Sosa A, Maamar E, Fernandez Martinez M, Ferjani S, Hammami S, et al. An Outbreak of NDM-1-Producing Klebsiella pneumoniae, Associated with OmpK35 and OmpK36 Porin Loss in Tunisia. Microb Drug Resist. 2018;24:1137-47.

12. Tsai YK, Fung CP, Lin JC, Chen JH, Chang FY, Chen TL, et al. Klebsiella pneumoniae outer membrane porins OmpK35 and OmpK36 play roles in both antimicrobial resistance and virulence. Antimicrob Agents Chemother. 2011;55(4):1485-93.

13. Soontaros S, Leelakanok N. Association between carbapenem-resistant Enterobacteriaceae and death: a systematic review and meta-analysis. Am J Infect Control. 2019;47:1200-12.

14. Karaiskos I, Lagou S, Pontikis K, Rapti V, Poulakou G. The "old" and the "new" antibiotics for MDR gram-negative pathogens: for whom, when, and how. Front Public Health. 2019;7:151

15. Zhang R, Liu L, Zhou H, Chan EW, Li J, Fang Y, et al. Nationwide surveillance of clinical Carbapenem-resistant Enterobacteriaceae (CRE) strains in China. EBioMedicine. 2017;19:98-106.

16. 2019 CaLSI. Performance Standards for Antimicrobial Susceptibility Testing CLSI,. 2019; M100-S25.(29th informational supplement.).

17. Dallenne C, Da Costa A, Decre D, Favier C, Arlet G. Development of a set of multiplex PCR assays for the detection of genes encoding important betalactamases in Enterobacteriaceae. J Antimicrob Chemother. 2010;65(3):490-5

18. Cattoir V, Poirel L, Rotimi V, Soussy CJ, Nordmann P. Multiplex PCR for detection of plasmid-mediated quinolone resistance qnr genes in ESBLproducing enterobacterial isolates. J Antimicrob Chemother. 2007;60(2):394-7.

19. Jacoby GA, Gacharna N, Black TA, Miller GH, Hooper DC. Temporal appearance of plasmid-mediated quinolone resistance genes. Antimicrob Agents Chemother. 2009;53(4):1665-6.

20. Poirel L, Walsh TR, Cuvillier V, Nordmann P. Multiplex PCR for detection of acquired carbapenemase genes. Diagn Microbiol Infect Dis. 2011;70(1):119-23.

21. Nakamura G, Wachino J, Sato N, Kimura K, Yamada K, Jin W, et al. Practical agar-based disk potentiation test for detection of fosfomycin- 
nonsusceptible Escherichia coli clinical isolates producing glutathione Stransferases. J Clin Microbiol. 2014;52(9):3175-9.

22. Diancourt L, Passet V, Verhoef J, Grimont PA, Brisse S. Multilocus sequence typing of Klebsiella pneumoniae nosocomial isolates. J Clin Microbiol. 2005; 43(8):4178-82.

23. Miyoshi-Akiyama T, Hayakawa K, Ohmagari N, Shimojima M, Kirikae T. Multilocus sequence typing (MLST) for characterization of Enterobacter cloacae. PLoS One. 2013;8(6):e66358.

24. Wang Q, Wang X, Wang J, Ouyang P, Jin C, Wang R, et al. Phenotypic and Genotypic Characterization of Carbapenem-resistant Enterobacteriaceae: Data From a Longitudinal Large-scale CRE Study in China (2012-2016). Clin Infect Dis. 2018;67(suppl_2):S196-s205.

25. Hemarajata P, Humphries RM. Ceftazidime/avibactam resistance associated with L169P mutation in the omega loop of KPC-2. J Antimicrob Chemother. 2019;74:1241-3

26. Huang W, Wang G, Sebra R, Zhuge J, Yin C, Aguero-Rosenfeld ME, et al. Emergence and Evolution of Multidrug-Resistant Klebsiella pneumoniae with both blaKPC and blaCTX-M Integrated in the Chromosome. Antimicrob Agents Chemother. 2017;61(7). https://doi.org/10.1128/AAC.00076-17.

27. Yu F, Hu L, Zhong Q, Hang Y, Liu Y, Hu X, et al. Dissemination of Klebsiella pneumoniae ST11 isolates with carbapenem resistance in integrated and emergency intensive care units in a Chinese tertiary hospital. J Med Microbiol. 2019;68(6):882-9.

28. Ito R, Mustapha MM. Widespread Fosfomycin Resistance in Gram-Negative Bacteria Attributable to the Chromosomal fosA Gene. mBio. 2017;8(4). https://doi.org/10.1128/mBio.00749-17.

29. Chen J, Wang D, Ding Y, Zhang L, Li X. Molecular Epidemiology of PlasmidMediated Fosfomycin Resistance Gene Determinants in Klebsiella pneumoniae Carbapenemase-Producing Klebsiella pneumoniae Isolates in China. Microb Drug Resist. 2019;25(2):251-7.

30. Priamchuk SD, Fursova NK, Anisimova VA, Kovalev lu N, Abaev IV, Kuzhel'naia EN, et al. An algorithm for identification of CTX-M-type betalactamase genes using restriction fragment length polymorphism analysis of PCR-product. Mol Gen Mikrobiol Virusol. 2011;(4):7-13.

31. Yuan M, Hall LM, Savelkoul PH, Vandenbroucke-Grauls CM, Livermore DM. SHV-13, a novel extended-spectrum beta-lactamase, in Klebsiella pneumoniae isolates from patients in an intensive care unit in Amsterdam. Antimicrob Agents Chemother. 2000:44(4):1081-4.

32. Kotsakis SD, Flach CF, Razavi M, Larsson DGJ. Characterization of the First OXA-10 Natural Variant with Increased Carbapenemase Activity. Antimicrob Agents Chemother. 2019;63(1).

33. Hammerum AM, Lester CH, Jakobsen L, Porsbo L. Faecal carriage of extended-spectrum beta-lactamase-producing and AmpC beta-lactamaseproducing bacteria among Danish army recruits. Clin Microbiol Infect. 2011; 17(4):566-8.

34. Carattoli A. Plasmids and the spread of resistance. Int J Med microbiol. 2013; 303(6-7):298-304.

35. Potter RF, D'Souza AW, Dantas G. The rapid spread of carbapenem-resistant Enterobacteriaceae. Drug Resist Updat. 2016;29:30-46.

36. Jing $Y$, Jiang $X$, Yin Z, Hu L, Zhang Y, Yang W, et al. Genomic diversification of IncR plasmids from China. J Global Antimicrob Resist. 2019;19:358-64.

37. Musicha P, Msefula CL, Mather AE, Chaguza C, Cain AK, Peno C, et al. Genomic analysis of Klebsiella pneumoniae isolates from Malawi reveals acquisition of multiple ESBL determinants across diverse lineages. J Antimicrob Chemother. 2019.74(5):1223-1232

38. Dobiasova H, Dolejska M. Prevalence and diversity of IncX plasmids carrying fluoroquinolone and beta-lactam resistance genes in Escherichia coli originating from diverse sources and geographical areas. J Antimicrob Chemother. 2016;71(8):2118-24.

39. Madec JY, Haenni M, Nordmann P, Poirel L. Extended-spectrum betalactamase/AmpC- and carbapenemase-producing Enterobacteriaceae in animals: a threat for humans? Clin Microbiol Infect. 2017;23(11):826-33.

40. Nordmann P, Poirel L, Walsh TR, Livermore DM. The emerging NDM carbapenemases. Trends Microbiol. 2011;19(12):588-95.

41. Wang Y, Tong MK, Chow KH, Cheng VC, Tse CW, Wu AK, et al. Occurrence of highly conjugative IncX3 epidemic plasmid carrying Bla NDM in Enterobacteriaceae isolates in geographically widespread areas. Front Microbiol. 2018:9:2272.

42. Jakobsen L, Bortolaia V, Bielak E, Moodley A, Olsen SS, Hansen DS, et al. Limited similarity between plasmids encoding CTX-M-1 beta-lactamase in
Escherichia coli from humans, pigs, cattle, organic poultry layers and horses in Denmark. J Glob Antimicrob Resist. 2015;3(2):132-6.

43. Carattoli A. Resistance plasmid families in Enterobacteriaceae. Antimicrob Agents Chemother. 2009:53(6):2227-38.

44. Bi D, Zheng J, Li JJ, Sheng ZK, Zhu X, Ou HY, et al. In Silico Typing and Comparative Genomic Analysis of IncFIIK Plasmids and Insights into the Evolution of Replicons, Plasmid Backbones, and Resistance Determinant Profiles. Antimicrob Agents Chemother. 2018;62(10). https://doi.org/10.1128/ AAC.00764-18.

\section{Publisher's Note}

Springer Nature remains neutral with regard to jurisdictional claims in published maps and institutional affiliations.
Ready to submit your research? Choose BMC and benefit from:

- fast, convenient online submission

- thorough peer review by experienced researchers in your field

- rapid publication on acceptance

- support for research data, including large and complex data types

- gold Open Access which fosters wider collaboration and increased citations

- maximum visibility for your research: over $100 \mathrm{M}$ website views per year

At BMC, research is always in progress.

Learn more biomedcentral.com/submissions 